\title{
Study on Characterization Method for Monitoring Data Analysis of Plane Displacement
}

\author{
WEI Shiyu ${ }^{1,2,3}$ a , JIANG Jun ${ }^{1,2,3, b *}$ and LI Chuan ${ }^{3, c}$ \\ ${ }^{1}$ Chongqing Engineering Research Center of Automatic Monitoring for Geological Hazards, \\ Chongqing 400042, China \\ ${ }^{2}$ Chongqing Institute of Geology and Mineral Resources, Chongqing 400042, China \\ ${ }^{3}$ Chongqing Huadi Design Institute of Engineering Investigation \\ aweishiyu.606@163.com, bjiangjun_dyy@163.com, ${ }^{c}$ 641419444@qq.com
}

\begin{abstract}
Keywords: plane displacement; monitoring; signal-noise separation; projection; characterization Abstract. In order to analyze and express the plane displacement vector of deformation monitoring more intuitively, confirm the value of deformation at the main deformation direction, and obtain the stabilization state of deformable body as well as the deformation development, an method named direct projection characterization method is proposed, on the basis of the fundamental theory of plane projection. The direct projection characterization method is an analytic method that projecting the plane deformation components onto the main deformation direction and calculating the deformation quantity. The engineering practice for monitoring indicates that this method is able to reflect the deformation vector at the main deformation direction more succinctly and intuitively. In addition, it can be applied to analyze the monitoring results of displacements for geological hazards including slopes, landslides, collapses, etc. It also can be used to serve the information feedback system and has an application value for engineering practice.
\end{abstract}

\section{Introduction}

The guiding ideology of geological hazard prevention is "prevention first and combining avoidance with control" "1]. As a result of the ideology, monitoring and early warning play an important role. Among those monitoring types, displacement monitoring is applied widely on account of its intuitive results and notable effect. In the engineering practice, the common method for plane displacement monitoring is geodetic surveying. And coordinate components under surveying coordinate system or their resultant vectors are used to characterize the deformation extent of deformable body in data processing. However, technicians pay more attention on the displacement along the main deformation direction of deformable body. Therefore, taking the safety monitoring data of a high slope in Chongqing reservoir of the Three Gorges as the research object, the error of displacement components is eliminated after confirming the main deformation direction. And the plane deformation components are projected onto the main deformation direction and the deformation quantity is calculated. Compared with the traditional characterization method, this analytic result is more accurate and more intuitive for reflecting the feature of plane displacements of slopes, and it is able to provide important data support for stability analysis and safety assessment of high slopes.

\section{Characterization method of plane displacement analysis}

At present, in the process of plane displacement monitoring for geological hazards, the traditional method, single component characterization method, has a problem that the real deformation vector and the data characterization vector do not tally. To solve this problem, the methods below are adopted.

(1)For displacement components, error eliminating and signal-noise separation are conducted to enhance the reliability of the one-dimensional components. 
(2) On the basis of the more reliable one-dimensional components, the deformation data is projected onto the known main deformation direction to characterize the deformation vectors.

Data processing of displacement components. During the deformation observation process, the observation data have three types of errors including gross error, systematical error and accidental error, causing by errors in observation environment, devices and workers. To ensure the reliability of displacement components data, the data processing flow is showed in Fig.1.

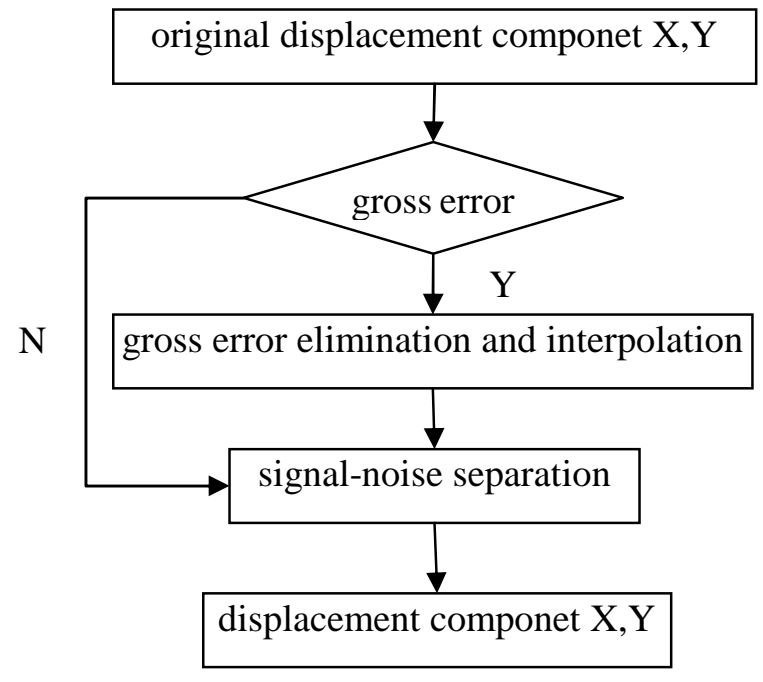

Fig. 1 Data processing flow of deformation components

Generally, the principle of " $3 \delta$ " can be used to judge whether the original observation sequence has gross error. After eliminating the gross error, algorithms like linear interpolation, Lagrange interpolation or polyfit can be adopted to interpolate the observation sequence to ensure the integrity of it in time domain. Then, curve fitting, multiple linear regression, $\operatorname{GM}(1,1)$, wavelet signal-noise separation and other mature algorithms can be used to process the data ${ }^{[2-8]}$.

Analytic characterization of projection. After error eliminating and signal-noise separation of plane displacement components, based on the more reliable displacement components, the azimuth of the main deformation direction of the slope is set as $\alpha$, and the displacement value of that projected onto the main glide direction (deformation rate or deformation accumulant) is expressed as $\Delta \mathrm{m}$. In addition, the displacement value of the direction from upslope towards downslope is positive. As shown in Fig.2, the projection values are expressed as equations below when the main direction is distributed in different quadrants.

$$
\begin{aligned}
& \Delta M=\Delta X \cos \alpha+\Delta Y \sin \alpha \\
& \Delta M=-\Delta X \cos \left(180^{\circ}-\alpha\right)+\Delta Y \sin \left(180^{\circ}-\alpha\right)=\Delta X \cos \alpha+\Delta Y \sin \alpha \\
& \Delta M=-\Delta X \cos \left(\alpha-180^{\circ}\right)-\Delta Y \sin \left(\alpha-180^{\circ}\right)=\Delta X \cos \alpha+\Delta Y \sin \alpha \\
& \Delta M=\Delta X \cos \left(360^{\circ}-\alpha\right)-\Delta Y \sin \left(360^{\circ}-\alpha\right)=\Delta X \cos \alpha+\Delta Y \sin \alpha
\end{aligned}
$$




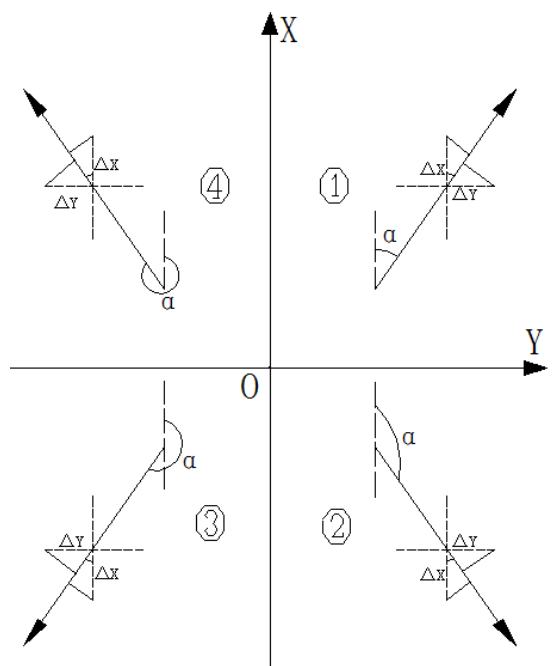

Fig. 2 projection analysis

From equations (1) to (4), displacement components of $\mathrm{X}$ and $\mathrm{Y}$ can be projected onto the main deformation direction when this direction is known. Then, the deformation process curve of monitoring points can be drawn by projection analytic values according to time sequence. As a result, the deformation feature of monitoring points can be characterized accurately and intuitively.

\section{Case study}

Taking the high slope in Chongqing reservoir of the Three Gorges as the example, horizontal displacements of the high slope are analyzed applying direct projection characterization method. That will be the reliable data support for slope stability assessment and information feedback.

Introduction of monitoring area. The elevation of the top of the researched high slope is $297 \mathrm{~m}$ to $299 \mathrm{~m}$ while that of the base of the slope is $214 \mathrm{~m}$ to $221 \mathrm{~m}$. The elevation difference is about $80 \mathrm{~m}$. The base of the slop is in the deep foundation excavation area, and the azimuth of main deformation direction $\alpha$ is 142 degree. Moreover, the length of the deformation area is $149 \mathrm{~m}$ and the width of it is $56 \mathrm{~m}$. Besides, the threat objects are the buildings for immigrants on the top of the slope. These buildings have 10 floors and are divided into three units with frame structure and pile foundation. There are 90 families, about 400 people, living in the buildings. Since November 2013, the grounds and walls in different floors have appeared different degrees of cracks, which caused that the security doors could not be closed. It has seriously affected the normal life of residents and became a threat to the safety of lives and properties of residents.

To eliminate the security risks altogether, the design units conducted emergency reinforcement design in highly deformed area (the security level is the highest). The principle of emergency reinforcement design is "dynamic design and informatization construction". Combining with the status quo of deformation development in monitoring area and the conditions of geological environment, plane displacement and subsidence observation, deep displacement monitoring, tilt and crack observation of buildings are applied to establish the comprehensive monitoring system. Meanwhile, pre-stressed anchor cable is adopted in the design for support. And whether to conduct compensate tension for anchor cable will be decided according to the feedback information of monitoring.

\section{Analytic characterization of monitoring data for plane displacement.}

Preprocessing of deformation components. The method of projection analytic characterization is analyzed for observed quantities of plane displacements. First, the original observed components are preprocessed to eliminate the gross error.

The plane displacements of this project are observed applying geodetic surveying by total station. The coordinate of the device center is set as $(\mathrm{X} 0, \mathrm{Y} 0, \mathrm{H} 0)$, the azimuth from the device center to the target point is $\beta$, and the horizontal distance from the center to the monitoring point is $\mathrm{D}$. The root 
mean square error of the plane coordinate components of monitoring points can be obtained by error propagation rate.

$$
\begin{aligned}
& m_{x}^{2}=\cos ^{2} \beta m_{D}^{2}+\frac{D^{2} \sin ^{2} \beta m_{\beta}^{2}}{\rho^{\prime \prime 2}} \\
& m_{Y}^{2}=\sin ^{2} \beta m_{D}^{2}+\frac{D^{2} \cos ^{2} \beta m_{\beta}^{2}}{\rho^{\prime \prime 2}}
\end{aligned}
$$

Taking the points (P6, P9, P11) on the principle monitoring section as the instances, the root mean square errors of coordinate components of monitoring points can be obtained by the equations (5) and (6), as shown in Table 1.

Table 1 The root mean square error of coordinate components

\begin{tabular}{ccccc}
\hline \multirow{2}{*}{$\begin{array}{c}\text { monitoring } \\
\text { point }\end{array}$} & azimuth & horizontal distance & \multicolumn{2}{c}{ root mean square error } \\
\cline { 3 - 4 } P6 & $301.4383^{\circ}$ & $200.262 \mathrm{~m}$ & $\mathrm{X}$ \\
P9 & $297.7467^{\circ}$ & $148.160 \mathrm{~m}$ & 0.94 & 1.00 \\
P11 & $298.7731^{\circ}$ & $166.566 \mathrm{~m}$ & 0.52 & 1.00 \\
\hline
\end{tabular}

According to the flow diagram in Fig.1, in the case of P6, the original deformation rate components are analyzed and processed as $3 \mathrm{X} \delta= \pm 2.82 \mathrm{~mm}, 3 \mathrm{Y} \delta= \pm 3.00 \mathrm{~mm}$. The phased achievements are shown in Fig.3.

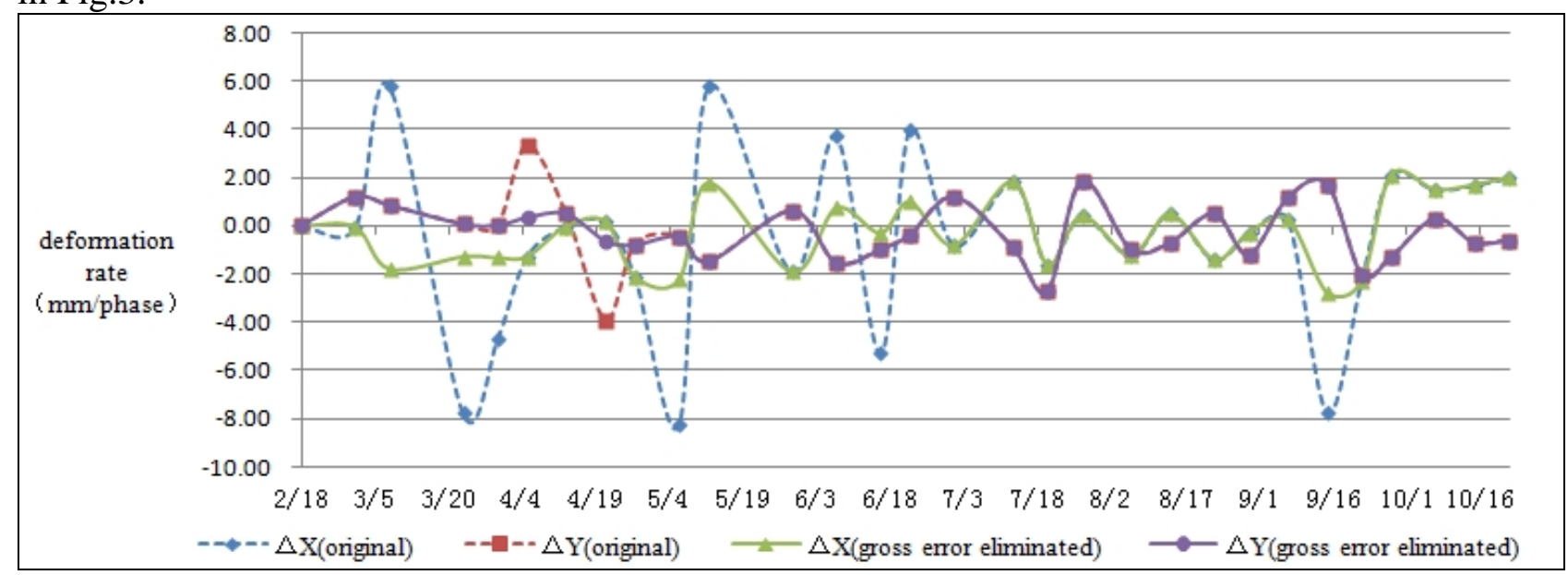

Fig. 3 deformation rate pretreatment(P6)

It can be seen from Fig.3 that the "jump" situation of the deformation rate components for P6 is inhibited. Thus, the results are capable to reflect the deformation rate of this point which is more close to the real situation.

Plane displacement analysis. Two methods are adopted to analytically characterize the displacement vectors taking P6 as the example $\left(\alpha=142^{\circ}\right)$. The first method is a traditional characterization method of displacement vectors under the surveying coordinate. The second method is the direct projection characterization method which is studied in this paper, and the result of it is shown in Table 2. 
Table 2 Comparison table of displacement vector characterization

\begin{tabular}{|c|c|c|c|c|c|}
\hline \multirow{2}{*}{$\begin{array}{l}\text { Monitoring } \\
\text { time }\end{array}$} & \multirow{2}{*}{$\Delta \Delta \mathbf{X}(\mathbf{m m})$} & \multirow{2}{*}{$\triangle \triangle \mathbf{Y}(\mathbf{m m})$} & \multicolumn{2}{|c|}{$\begin{array}{c}\text { The first chracterization } \\
\text { method }\end{array}$} & \multirow{2}{*}{$\begin{array}{l}\text { The second } \\
\text { chracterization } \\
\text { method ( } \mathbf{m m})\end{array}$} \\
\hline & & & diplacement (mm) & $\operatorname{azimuth}\left(^{\circ}\right)$ & \\
\hline $2 / 18$ & 0.00 & 0.00 & 0.00 & & 0.00 \\
\hline $3 / 1$ & -0.05 & 1.20 & 1.20 & 92.44 & 0.78 \\
\hline $3 / 8$ & -1.84 & 2.05 & 2.75 & 131.98 & 2.71 \\
\hline $3 / 23$ & -3.12 & 2.12 & 3.77 & 145.87 & 3.76 \\
\hline $3 / 30$ & -4.44 & 2.13 & 4.93 & 154.35 & 4.81 \\
\hline $4 / 5$ & -5.72 & 2.46 & 6.23 & 156.68 & 6.02 \\
\hline $4 / 13$ & -5.74 & 2.96 & 6.46 & 152.69 & 6.35 \\
\hline $4 / 21$ & -5.57 & 2.33 & 6.04 & 157.27 & 5.83 \\
\hline $4 / 27$ & -7.68 & 1.55 & 7.84 & 168.61 & 7.01 \\
\hline $5 / 6$ & -9.91 & 1.11 & 9.97 & 173.61 & 8.49 \\
\hline $5 / 12$ & -8.14 & -0.37 & 8.15 & 182.59 & 6.19 \\
\hline $5 / 29$ & -10.03 & 0.25 & 10.04 & 178.54 & 8.06 \\
\hline $6 / 7$ & -9.30 & -1.28 & 9.39 & 187.86 & 6.54 \\
\hline $6 / 16$ & -9.60 & -2.27 & 9.87 & 193.27 & 6.17 \\
\hline $6 / 22$ & -8.62 & -2.63 & 9.01 & 196.99 & 5.17 \\
\hline $7 / 1$ & -9.42 & -1.43 & 9.53 & 188.62 & 6.54 \\
\hline $7 / 13$ & -7.59 & -2.34 & 7.94 & 197.17 & 4.54 \\
\hline $7 / 20$ & -9.20 & -5.06 & 10.50 & 208.80 & 4.14 \\
\hline $7 / 27$ & -8.79 & -3.20 & 9.36 & 200.02 & 4.96 \\
\hline $8 / 6$ & -10.02 & -4.16 & 10.85 & 202.55 & 5.33 \\
\hline $8 / 14$ & -9.52 & -4.86 & 10.69 & 207.05 & 4.51 \\
\hline $8 / 23$ & -10.92 & -4.36 & 11.76 & 201.77 & 5.92 \\
\hline $8 / 30$ & -11.22 & -5.56 & 12.53 & 206.37 & 5.42 \\
\hline $9 / 7$ & -10.96 & -4.39 & 11.81 & 201.82 & 5.94 \\
\hline $9 / 15$ & -13.72 & -2.68 & 13.98 & 191.05 & 9.16 \\
\hline $9 / 22$ & -16.02 & -4.68 & 16.69 & 196.29 & 9.74 \\
\hline $9 / 28$ & -13.92 & -5.98 & 15.15 & 203.25 & 7.29 \\
\hline $10 / 7$ & -12.42 & -5.68 & 13.66 & 204.58 & 6.29 \\
\hline $10 / 15$ & -10.72 & -6.38 & 12.47 & 210.76 & 4.52 \\
\hline $10 / 22$ & -8.72 & -6.98 & 11.17 & 218.68 & 2.57 \\
\hline
\end{tabular}

At present, the first characterization method is widely used. Nevertheless, the characterization process includes displacement coordinate calculation, displacement quadrant calculation and displacement azimuth matrixing while the characterization results have to be assisted by displacement azimuth and drawings to express intuitively. Based on the projection principle which is same to the first characterization method, the components are directly projected onto the main deformation direction with resultant calculation. Thus, this type of analytic calculation is more simple and the result of it is more intuitively. As shown in Fig.4, the deformation development trend of monitoring points can be determined by the process line of displacement vectors of the main deformation direction. 


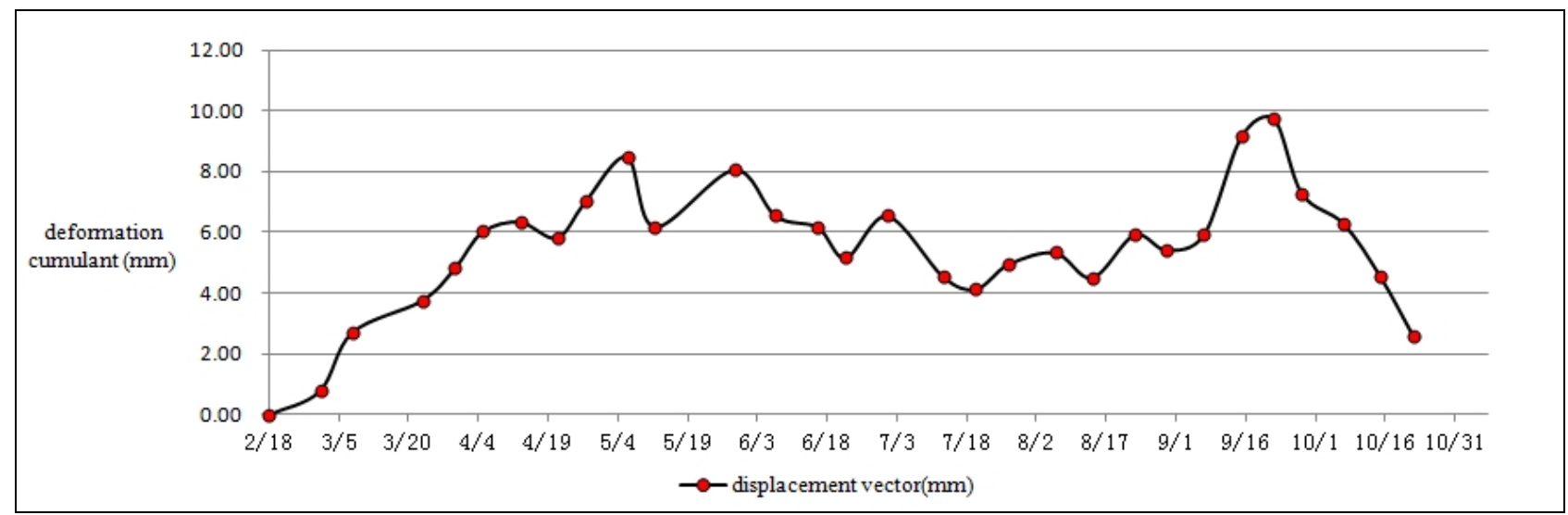

Fig. 4 Cumulative deformation process of points

It can be seen from Fig.4 that the plane displacements of P6 has experienced three stages. The first stage was from 18 February 2014 to 6 May 2014. The anchor cables of the treatment project have not been tensioned. The tangential angle of deformation rate for this point $\alpha$ became smaller and the slope was in the initial deformation stage. The second stage is divided into two phases. The first phase is from 6 May 2014 to 3 August 2014. In this phase, the anchor cable began to be tensioned and the monitoring data indicated that the deformation trend was restrained and the slope tended to be stable. The second phase is from 3 August 2014 to 22 September 2014. This phase was impacted by the rare heavy rain in the northeast of Chongqing. The deformation rate increased unexpectedly. The relevant information was fed back to the design and construction units immediately and the designers decided to construct the second row of anchor cables. The three stage is from 22 September 2014 to 22 October 2015. This stage is the period of tension construction for anchor cables. The monitoring data showed that the deformation developed towards the negative direction, which explained that the expected results were obtained by anchor cable construction and the accelerated deformation trend was removed.

\section{Conclusion}

In the field of geological hazard, ground displacement monitoring is the most common and effective monitoring method. At present, the traditional method, resultant of displacement components, is widely used by techniques. The displacement vectors are characterized by displacement scalar and displacement azimuth. The disadvantages of this method is the complex calculation and analysis, and it is failed to obtain the intuitive results. In the case of a high slope in Chongqing reservoir in the Three Gorges, the direct projection characterization method is discussed in this paper. After gross error elimination and single-noise separation of the observed displacement components, the deformation components are directly projected onto the main deformation direction to characterize the displacement conditions of monitoring points along the main deformation direction. The practice proves that applying the direct projection characterization method turns calculation and analysis to be more simple and efficient and makes characterization more reasonable and intuitive. It is able to serve the system "dynamic design and informatization construction" better and has an application value for engineering practice.

\section{References}

[1] Order of the State Council of the people's Republic of China, the 394th order. Regulations of geological disaster prevention and control[S].2004.

[2] He Kai, Yin Yueping, Li Bin. Analysis of characteristics and stability for Zengziyan - Eryayan unstable rocks belt in Nanchuan county Chongqing[J]. The Chinese Journal of Geological Hazard and Control, 2015, 01:16-22. 
[3] He Zhaoyang, Ju Nengpan, Huang Jian. Research on the processing method of big data from geo-hazard monitoring[J]. Safety and Environmental Engineering, 2014,05:115-120.

[4] He Huaifeng, Li Minglu, Liu Junzhu. Application of wavelet analysis in steep seem mining subsidence monitoring data analysis[J]. The Chinese Journal of Geological Hazard and Control, 2014,04:76-80.

[5] Tang Hongmei, Wei Lai, Tang Yunhui, et al. Correlation analysis and prediction model for rainfall-induced landslide in Chongqing area[J]. The Chinese Journal of Geological Hazard and Control, 2013,04:16-22.

[6] Yue Jianping, Fang Lu, Li Ni. The study on the data processing by using the time series analysis methods for building settlement observation[J]. Science of Surveying and Mapping, 2007(7):1-4.

[7] Chen Zhengyao, Li Shihai, Feng Chun. Stability analysis about slope based on strain strength distribution model[J]. The Chinese Journal of Geological Hazard and Control, 2014,04:11-17.

[8] Ju Chunyan, Wang Zhiliang, Liu Zhen, et al. Analysis of geological disaster deformation monitoring in Laoshan district, Qingdao[J]. Journal of Engineering Geology, 2013,03:450-458. 